\title{
Finite Element Analysis of Oil and Gas Field Development Completion Tool Based on ANSYS
}

\author{
Yanli Liu ${ }^{1}$, Daqi Fu ${ }^{2}$, Gaofeng Zhang ${ }^{1}$, Yanzheng Yang ${ }^{1}$, Haijun Zhang ${ }^{1}$, Haiwei Li $^{3}$ \\ ${ }^{1}$ Petroleum Engineering Research Institute, PetroChina Dagang Oilfield Company, Tianjin, 300280, China \\ ${ }^{2}$ Engineering Technology and supervision Department, PetroChina Dagang Oilfield Company, Tianjin, 300280, China \\ ${ }^{3}$ Fourth Oil Production Plant (Beach Development Company), PetroChina Dagang Oilfield Company, Tianjin, 300280, China
}

\begin{abstract}
At persent, multi expandable patching can't be operated below the patched period of casing damage wells, a varible diameter expandable cone has been designed, so expandable tools can be put into wells with small diameter and expanded with large diameter which can achieve the purpose of patching for casing damage wells for many times. The indoor prototype testing showed that the tools had a suitable structure, start-up pressure was 3-5MPa,complete closure pressure was 52MPa,maximum overall diameter increased from $188 \mathrm{~mm}$ to $220 \mathrm{~mm}$, with the same size of conventional expandable cone, expandable rate was $17.02 \%$. The research results showed that expandable force increases linearly with the increase of thrust, expandable tools could be put into wells with small diameter and expanded with large diameter with the help of variable diameter expandable cone, it should have a broad application prospect after rudcing the closure pressure.
\end{abstract}

\section{Introduction}

Expandable pipe patching technology appeared in the late 1980s, was invented by a Shell Company, it was mainly used in fixing damaged casing leakage, plugging perforation casing, lateral drilling completion, plugging drilling complex strata, etc.. Dagang Oilfield is in the middle and late stage of development, and the casing damage is more common. Currently, the number of registered casing damage Wells accounts for $19.45 \%$ of the total oil Wells in the field. Expandable tube single subsidies technology was used 30 Wells in dagang oilfield nearly three years, but if the new points of casing damage was in the expandable patching pipe below, now secondary patching or patching for many times can't be used, when severe cases it will be abandoned well. Therefore, a variable diameter expandable cone is urgently needed to realize patching for many times of casing damage well. The diameter of expandable tool is small during into well, a large diameter will be obtained during patching process ${ }^{[1]-[7]}$. The basic structure of variable diameter expandable tool was studied by Tang xingbo et al. and the wedge Angle and length were optimized.

\section{The principle of variable diameter expandable cone}

The conventional expandable cone is pre-assembled in the expandable pipe, and only one patching can be used for the same damaged well. When the casing damage is below the patched period of casing damage wells, because the inner diameter has is smaller, conventional expandable cone cannot pass, so the design ideas, ideas and basic principles of the variable diameter expandable cone should be studied.

The design of variable diameter expandable wedge block was mainly concerned with three parameters, wedge length, wedge angle and thickness. The longer the wedge length and the larger the wedge angle, the larger the change in the outer diameter of the expandable cone. However, the greater the length of the wedge block, the greater the probability of interference between adjacent wedge blocks. The larger the wedge angle, the larger the corresponding expandable thrust. The thicker the wedge thickness, the higher the strength, but the probability of interference between adjacent wedge blocks increased, and the size of the center tube was limited. The single expandable cone block was assembled through the handle to prevent the expandable cone from falling off. The assembly structure is shown in Figure.1.

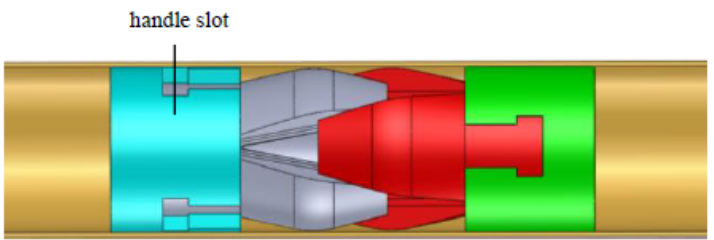

(a) before closing 


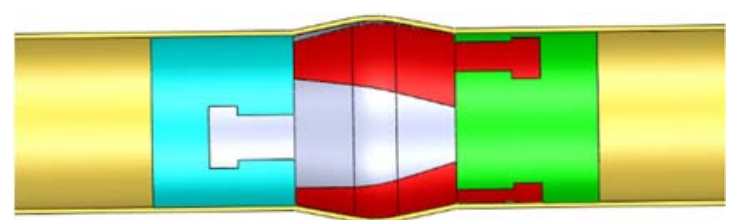

(b) after closing

Figure.1 Variable diameter expandable cone structure before and after closing

In order to avoid the dislocation of the adjacent expandable cone block in the running process, the complete expandable cone can not be formed, so a concave and convex mating orbits were designed on the two sides of the expandable cone block. Solid Works software was Used for expandable cone $3 \mathrm{~d}$ assembly and simulation movement, the results show that the design of variable diameter expandable cone was good, no interference phenomenon, could be put into the expandable pipe smoothly. The final design of the expandable cone thickness was $27.0 \mathrm{~mm}$, the largest was $43.0 \mathrm{~mm}$, the total length was $300.0 \mathrm{~mm}$, the outer diameter before change was $188.0 \mathrm{~mm}$, and the outer diameter after change was $220.0 \mathrm{~mm}$. The total distance of the expandable cone is $90.0 \mathrm{~mm}$, and the thrust surface and thrust direction of the expandable cone were vertical after the position was in place, so the phenomenon of "external splitting" was avoided after the expandable cone was in place.

\section{The finite element analysis of variable diameter expandable cone}

The mechanical properties and motion characteristics of the variable diameter expandable cone were analyzed, which verified that the structure design was feasible in theory, and some instructive suggestions were given for the design of the friction pairs. The variable diameter expandable cone structure was complex and stresses were more. In order to obtain the internal stress and strain, finite element analysis was necessary to further optimize its structure.

\section{1. the mathematical model of finite element analysis}

The parameters of stress, strain and displacement was got by the finite element analysis. Equivalent stress was determined by the von Mises yield criterion:

$$
\sigma_{e q}=\sqrt{\frac{1}{2}\left[\left(\sigma_{1}-\sigma_{2}\right)^{2}+\left(\sigma_{2}-\sigma_{3}\right)^{2}+\left(\sigma_{3}-\sigma_{1}\right)^{2}\right]}
$$

In the formula: $\sigma_{1}, \sigma_{2}$ and $\sigma_{3}$ were the main stresses, $\mathrm{MPa}$; And $\sigma_{1}>\sigma_{2}>\sigma_{3}$.

The material failed when $\sigma_{e q}>[\sigma]$.

Displacement analysis, according to the relationship between strain and displacement in linear elastic mechanics: $\varepsilon_{x}=\partial \mu / \partial x, \varepsilon_{y}=\partial v / \partial y$,

$$
\begin{aligned}
& \varepsilon_{x y}=\partial \mu / \partial x+\partial \boldsymbol{v} / \partial y \text {, we got: } \\
& \varepsilon=\left[\begin{array}{l}
\varepsilon_{x} \\
\varepsilon_{y} \\
\varepsilon_{x y}
\end{array}\right]=\left[\begin{array}{c}
\partial \mu / \partial x \\
\partial v / \partial y \\
\partial \mu / \partial x+\partial v / \partial y
\end{array}\right]=\left[\begin{array}{cc}
\partial / \partial x & 0 \\
0 & \partial / \partial y \\
\partial / \partial x & \partial / \partial y
\end{array}\right]\left[\begin{array}{l}
\mu \\
v
\end{array}\right] \\
& =\frac{1}{2 A}\left[\begin{array}{cccccc}
b_{i} & 0 & b_{j} & 0 & b_{k} & 0 \\
0 & c_{i} & 0 & c_{j} & 0 & c_{k} \\
c_{i} & b_{i} & c_{j} & b_{j} & c_{k} & b_{k}
\end{array}\right]\left[\begin{array}{c}
\mu_{i} \\
v_{i} \\
\mu_{j} \\
v_{j} \\
\mu_{k} \\
v_{k}
\end{array}\right]=\frac{1}{2 A}\left[\begin{array}{c}
b_{i} \mu_{i}+b_{j} \mu_{j}+b_{k} \mu_{k} \\
c_{i} v_{i}+c_{j} v_{j}+c_{k} v_{k} \\
b_{i} \mu_{i}+b_{j} \mu_{j}+b_{k} \mu_{k}+c_{i} v_{i}+c_{j} v_{j}+c_{k} v_{k}
\end{array}\right]
\end{aligned}
$$

In the formula: $\varepsilon$ was the strain, $\mathrm{m}, \mathrm{A}$ was the crosssectional area, $\mathrm{m} 2, \mu, \mathrm{v}$ were the displacement of $\mathrm{x}, \mathrm{y}$ direction, $\mathrm{m}, \mathrm{b}$ and $\mathrm{c}$ are the displacement of the unit node, $\mathrm{m}$.

Based on the above mathematical model, the finite element analysis of the variable diameter expandable cone was carried out, and the weak links were improved according to the stress analysis results to meet the strength requirements.

\section{2. geometric model and grid division.}

Using SolidWorks 3d drawing software, the geometric model of variable diameter expandable cone was established, and the model was imported into ANSYS Workbench finite element analysis software and grid division was carried out. Tetrahedron structure were maily used in Meshing, division of grid had a total of 3007 nodes, 1603 units, the average unit quality was 0.67 , grid average aspect ratio was 2.5 , parallel deviation of average was 0 , deviation average was 0.47 , orthogonal quality averages was 0.73 , the expandable cone mesh quality was good, accord with the requirement of finite element analysis.

\section{3. the material properties define and apply load}

42CrMo super-strength alloy structural steel were used in the expandable cone material, material elastic modulus was $203 \mathrm{GPa}$, poisson ratio was 0.33 , density was 7.85 $\mathrm{g} / \mathrm{cm} 3$, tensile strength was $1080 \mathrm{MPa}$, yield strength was $930 \mathrm{MPa}$. The friction force, expandable pipe binding force and extrusion pressure were loaded, and the free of degree at the end of the handle was set to 0 , and the phenomenon of cold welding and blocking during the operation of the expandable cone was simulated.

\section{4. solution and result analysis}

The stress distribution cloud diagram of the expandable cone was obtained by finite element analysis, as shown in Figure.8.Maximum stress value was in the middle of the handle position, $822.6 \mathrm{MPa}$, the value was close to 930.0 MPa (The 42 CrMo material yield strength), the stress concentration phenomenon was very obvious, serious deformation and even broken may occur for variable diameter expandable cone handle in the process of movement. Therefore, the structure of the expandable cone needed to be optimized to reduce the stress concentration. 


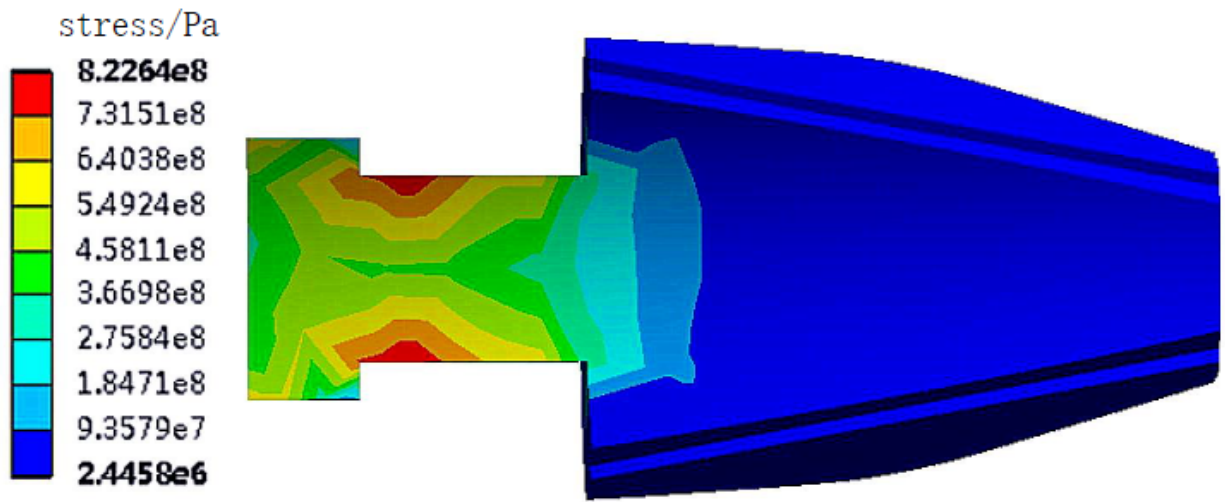

Figure.2 The stress distribution of variable diameter expandable cone

\section{5. structure optimization of the variable diameter expandable cone}

3.5.1. Round corner treatment. The rounded corner treatment was an effective means to reduce stress concentration, and the rounded angle (radius $3.0 \mathrm{~mm}$ ) was made on all edges of the handle to the stress concentration of the structure. The circular angle (radius $2.0 \mathrm{~mm}$ ) was made on the expandable cone body edge, and the relative motion of the two concave and convex expandable cones was made to avoid the scraping injury of the sharp angle.

3.5.2. Inclination on the handle. $10^{\circ}$ inclination was made for handle, so axial and radial displacement could be produced smoothly by expandable cone block, the radial friction was reduced, the expandable process was more smooth. The size deviation of the handle was designed as $-0.5 \sim-1.0 \mathrm{~mm}$, and the size deviation of the handle slot was designed to be $0.5-1.0 \mathrm{~mm}$, so that the handle had enough space for activity.
3.5.3. The thick handle. Through $3 \mathrm{~d}$ assembly interference analysis, found that when two adjacent concave or convex cone expandable block interference occured, the distance between the inside of the circular arc surface of handle and the center tube cylindrical surface was $10.0 \mathrm{~mm}$, the inside of the handle could be thicken appropriately to enhance mechanical strength.

\section{6. finite element mechanical analysis after structural optimization}

The finite element mechanical analysis was made for the expandable cone of optimized structure, and the results were shown in Figure.9. The maximum stress distribution position was shifted to the end of the handle by $5.0 \mathrm{~mm}$, and the working condition of the tool was improved. The maximum stress value was $454 \mathrm{MPa}$, which was far less than the yield strength of the material, which met the requirement of material strength and was safe and reliable. After optimization, the stress distribution of the expandable cone was more uniform and the transition was more moderate.

stress/Pa
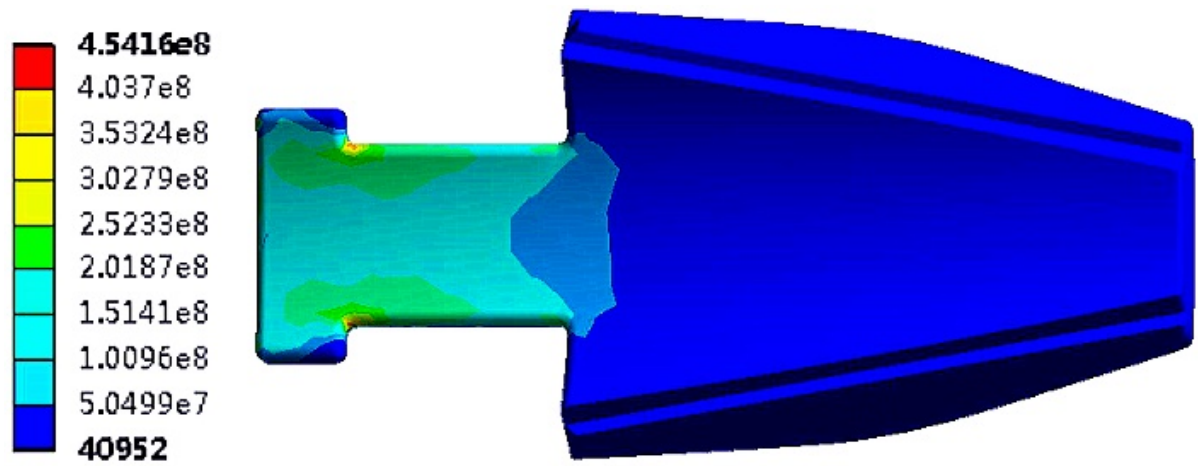

Figure.3 The stress distribution of variable diameter expandable cone after structure optimization

\section{4 laboratory prototype test}

To verify the correctness of the mechanism and the rationality of the structure design of the variable diameter expandable cone, a set of variable diameter expandable tools prototype was processed, the indoor test of the variable diameter expandable cone closing process and the hydraulic seal compression performance were made.

Test results show that the variable diameter expandable cone could start and close successfully, the start-up pressure of expandable cone was 3 - $5 \mathrm{MPa}$, expandable cone closure pressure was $52 \mathrm{MPa}$, the maximum diameter of expandable cone increase from $188.0 \mathrm{~mm}$ to $188.0 \mathrm{~mm}$ after closed completely, expandable rate was $17.02 \%$, the expandable pipe diameter increased from $203.0 \mathrm{~mm}$ to $231.8 \mathrm{~mm}$, expandable rate was $15.06 \%$.The experimental results were consistent with structural design, mechanical analysis, motion analysis, surface treatment and finite element analysis, which laied 
a foundation for the next field test and application. At the same time, in the test we found that completely closed pressure of expandable cone was too high, so multi-stage pressurization mechanism would be designed next step, the closed completely pressure of expandable cone would be controled in $18-20 \mathrm{MPa}$, matched with the site operation pressure.

\section{5 conclusion}

5.1. The size and structure design of variable diameter expandable cone are reasonable, interference between components wouldn't occur during operation, and the design conforms to the requirements of mechanical law and motion law. The expandable cone can run smoothly, and there is no friction self-locking between tools, which can realize the function of small diameter into well and large diameter expandable.

5.2. When the thrust size is certain, the more smooth the adjacent contact surface of variable diameter expandable cone block is, the smaller the coefficient of friction, the bigger expandable force, the easier it is to overcome the expandable pipe binding to implement expansion. When the expandable tube material is determined, the smaller the friction coefficient of the expandable cone end, the smoother the surface, the smaller the required thrust, the lower the requirement of the hydraulic system.

\section{Acknowledgments}

Dagang Oilfield Company scientific research project "Large displacement long horizontal Well gravel pack completion Technology Research and Application" part of the research content.

\section{References}

1. MERRITT R M, GUSEVIK R, BUCKLER W, et al. Well remediation using expandable cased-hole liners[J]. World Oil,2002,223(7): 56-65.

2. MA Hongtao, JI Changjie. The development and application of expandable tubular technique[J]. Foreign Oil Field Engineering, 2006,22(2): 20-24.

3. TANG Xingbo, LI Qian, LIU Yonggang. Optimized design of variable diameter expandable Tool for expandable tubular[J]. Oil Field Equipment, 2008, 37(11): 23-25.

4. ZHENG Tao. Diameter taper variable structure optimization and mechanics analysis[D]. Qingdao: China University of Petroleum(Huadong),2014.

5. CHEN Peiliang,JING Enjiang,WANG Yuduo,et al. Drilling and expandable casing completion for complex formation isolation in sidetrack well[J].China Petroleum Machinery, 2015, 43(12): 25-28.

6. LI Tao. Solid expandable tubular patching technique for high-temperature and high-pressure casing damaged wells[J]. Petroleum Exploration and Development, 2015, 42(3): 374-378.

7. WEI Songbo, PEI Xiaohan, SHI Bairu, et al. Wear resistance and anti-friction of expandable cone with hard coating $[\mathrm{J}]$. Petroleum Exploration and Development, 2016, 43(2): 297-302. 